\title{
The expanding clinical phenotype of Bosch-Boonstra-Schaaf optic atrophy syndrome: 20 new cases and possible genotype-phenotype correlations
}

\author{
Chun-An Chen, MS ${ }^{1,2}$, Daniëlle G.M. Bosch, MD, PhD ${ }^{3-6}$, Megan T. Cho, ScM, CGC7, \\ Jill A. Rosenfeld, MS, CGC ${ }^{1}$, Marwan Shinawi, MD ${ }^{8}$, Richard Alan Lewis, MD, MS ${ }^{1,9}$, John Mann, MD ${ }^{10}$, \\ Parul Jayakar, MD, MS ${ }^{11}$, Katelyn Payne, RN, CGC ${ }^{12}$, Laurence Walsh, MD ${ }^{12,13}$, \\ Timothy Moss, MD, PhD ${ }^{14}$, Allison Schreiber, MS, LGC ${ }^{14}$, Cheri Schoonveld, MS, CGC ${ }^{15}$, \\ Kristin G. Monaghan, $\mathrm{PhD}^{7}$, Frances Elmslie, $\mathrm{MD}^{16}$, Ganka Douglas, $\mathrm{PhD}^{7}$, \\ F. Nienke Boonstra, MD, PhD ${ }^{4,6}$, Francisca Millan, MD', Frans P.M. Cremers, PhD ${ }^{3,5}$, \\ Dianalee McKnight, $\mathrm{PhD}^{7}$, Gabriele Richard, MD7, Jane Juusola, PhD7 , Fran Kendall, MD ${ }^{17,18}$, \\ Keri Ramsey, RN ${ }^{19}$, Kwame Anyane-Yeboa, $\mathrm{MD}^{20}$, Elfrida Malkin, MD, $\mathrm{MPH}^{21}$, \\ Wendy K. Chung, MD, PhD²0,22, Dmitriy Niyazov, MD²3, Juan M. Pascual, MD, PhD24, \\ Magdalena Walkiewicz, PhD1, Vivekanand Veluchamy, MD ${ }^{25}$, Chumei Li, MD, PhD²6, \\ Fuki M. Hisama MD27, Bert B.A. de Vries, MD, PhD ${ }^{3,6}$ and Christian Schaaf, MD, PhD 1,2,28
}

Purpose: Bosch-Boonstra-Schaaf optic atrophy syndrome (BBSOAS) is an autosomal-dominant disorder characterized by optic atrophy and intellectual disability caused by loss-of-function mutations in NR2F1. We report 20 new individuals with BBSOAS, exploring the spectrum of clinical phenotypes and assessing potential genotype-phenotype correlations.

Methods: Clinical features of individuals with pathogenic NR2F1 variants were evaluated by review of medical records. The functional relevance of coding nonsynonymous $N R 2 F 1$ variants was assessed with a luciferase assay measuring the impact on transcriptional activity. The effects of two start codon variants on protein expression were evaluated by western blot analysis.

Results: We recruited 20 individuals with novel pathogenic NR2F1 variants (seven missense variants, five translation initiation variants,

\section{INTRODUCTION}

Bosch-Boonstra-Schaaf optic atrophy syndrome (BBSOAS) was recently identified as an autosomal-dominant disorder characterized by optic atrophy, developmental delay, and intellectual two frameshifting insertions/deletions, one nonframeshifting insertion/deletion, and five whole-gene deletions). All the missense variants were found to impair transcriptional activity. In addition to visual and cognitive deficits, individuals with BBSOAS manifested hypotonia (75\%), seizures (40\%), autism spectrum disorder (35\%), oromotor dysfunction (60\%), thinning of the corpus callosum $(53 \%)$, and hearing defects (20\%).

Conclusion: BBSOAS encompasses a broad range of clinical phenotypes. Functional studies help determine the severity of novel NR2F1 variants. Some genotype-phenotype correlations seem to exist, with missense mutations in the DNA-binding domain causing the most severe phenotypes.

Genet Med advance online publication 17 March 2016

Key Words: BBSOAS; developmental delay; NR2F1; optic atrophy

disability (OMIM 615722). ${ }^{1}$ BBSOAS results from mutations in $N R 2 F 1$, which encodes a conserved orphan nuclear receptor and transcriptional regulator. ${ }^{2}$ The ligand of NR2F1 remains unknown. Similar to most nuclear receptors, NR2F1 contains

\footnotetext{
The first two authors and the last two authors contributed equally to this work, and the last two authors are co-senior authors.

${ }^{1}$ Department of Molecular and Human Genetics, Baylor College of Medicine, Houston, Texas, USA; ${ }^{2}$ Jan and Dan Duncan Neurological Research Institute, Texas Children's Hospital, Houston, Texas, USA; ${ }^{3}$ Department of Human Genetics, Radboud University Medical Center, Nijmegen, The Netherlands; ${ }^{4}$ Bartiméus, Institute for the Visually Impaired, Zeist, The Netherlands; ${ }^{5}$ Radboud Institute for Molecular Life Sciences, Radboud University Medical Center, Nijmegen, The Netherlands; ${ }^{6}$ Department of Cognitive Neuroscience, Donders Institute for Brain, Cognition and Behaviour, Radboud University Medical Center, Nijmegen, The Netherlands; ${ }^{7}$ GeneDx, Gaithersburg, Maryland, USA; ${ }^{8}$ Division of Genetics and Genomic Medicine, Department of Pediatrics, Washington University School of Medicine, St. Louis, Missouri, USA; ${ }^{9}$ Department of Ophthalmology, Baylor College of Medicine, Houston, Texas, USA; ${ }^{10}$ Genetics, Kaiser-Permanente Fresno Medical Center, Clovis, California, USA; ${ }^{11}$ Nicklaus Children's Hospital, Miami, Florida, USA; ${ }^{12}$ Riley Hospital for Children, Indianapolis, Indiana, USA; ${ }^{13}$ Departments of Neurology, Medical and Molecular Genetics, and Pediatrics, Indiana University School of Medicine, Indianapolis, Indiana, USA; ${ }^{14}$ Genomic Medicine Institute, Cleveland Clinic, Cleveland, Ohio, USA; ${ }^{15}$ University of Minnesota Health, Minneapolis, Minnesota, USA; ${ }^{16}$ South West Thames Regional Genetics Service, St. George's Healthcare NHS Trust, London, UK; ${ }^{17}$ VMP Genetics, LLC, Atlanta, Georgia, USA; ${ }^{18}$ University of Georgia, Athens, Georgia, USA; ${ }^{19}$ Center for Rare Childhood Disorders, Translational Genomics Research Institute, Phoenix, Arizona, USA; ${ }^{20}$ Department of Pediatrics, Columbia University Medical Center, New York, New York, USA; ${ }^{21}$ Nyack Hospital, Nyack, New York, USA; ${ }^{22}$ Department of Medicine, Columbia University Medical Center, New York, New York, USA; ${ }^{23}$ Division of Medical Genetics, Department of Pediatrics, Ochsner Clinic Foundation, New Orleans, Louisiana; ${ }^{24}$ Department of Neurology and Neurotherapeutics, University of Texas Southwestern Medical Center, Dallas, Texas, USA; ${ }^{25}$ Division of Pediatric Neurology, Akron Children's Hospital, Akron, Ohio, USA; ${ }^{26}$ McMaster University Medical Center, Hamilton, Ontario, Canada; ${ }^{27}$ Department of Medicine, Division of Medical Genetics, University of Washington, Seattle, Washington, USA; ${ }^{28}$ Texas Children's Hospital, Houston, Texas, USA. Correspondence: Bert B.A. de Vries and Christian P. Schaaf (Bert.deVries@radboudumc.nl; Schaaf@bcm.edu)
} 
a DNA-binding domain (DBD), formed by two zinc-finger domains, and a putative ligand-binding domain. ${ }^{3}$ The physiological functions of NR2F1 have been studied extensively thanks to an Nr2f1 knockout mouse model (the human NR2F1 and murine Nr2f1 proteins share $99 \%$ amino acid identity). Previous studies showed that $\mathrm{Nr} 2 \mathrm{fl}$ is important for cortical patterning, ${ }^{4-7}$ thalamocortical axon guidance, ${ }^{8}$ neurogenesis, ${ }^{8}$ arborization, ${ }^{9}$ and eye and optic nerve development. ${ }^{10,11}$ By contrast, studies of human disorders resulting from loss-of-function NR2F1 mutations have been limited. ${ }^{1,12,13}$ Moreover, these studies may not be sufficient to describe the full phenotypic spectrum of BBSOAS because of the small number of cases identified so far. Here we report 20 new individuals with pathogenic NR2F1 variants and summarize their clinical features.

\section{Human subjects}

\section{MATERIALS AND METHODS}

Individuals with probable pathogenic NR2F1 variants were enrolled on the basis of their genotypes. From clinical wholeexome sequencing data, we selected coding nonsynonymous variants that were not present in the ESP5400 Exome Variant Server and that were predicted to be damaging/probably damaging by at least one algorithm (PolyPhen-2/Mutation Taster). All these were confirmed by Sanger sequencing. Whole-gene deletion mutations were identified based on clinical chromosome microarray analysis. Three individuals (individuals 1, 3, and 11) were enrolled through Baylor Miraca Genetics Laboratories and eight (individuals 2, 5, 7, 8, 10,12, 14, and 15) through the GeneDx laboratory. Upon identification of a probable pathogenic variant in $N R 2 F 1$, genetics counselors from the respective laboratories contacted the referring providers to inform them of research interest by Dr Schaaf's laboratory. Four other individuals (individuals 9, 16,17, and 18) were enrolled through the Radboud University Medical Center, Nijmegen, the Netherlands. Individual 9 has been reported before ${ }^{14}$ and re-analysis of the whole-exome sequencing data showed a low covered de novo NR2F1 variant, which was validated by Sanger sequencing. The referring providers of individuals 16,17 , and 18 contacted Dr de Vries. For the rest of the individuals (individuals 4, 6, 13, 19, and 20 ), the referring provider or family contacted Dr Schaaf directly because of a new diagnosis of BBSOAS made by genetic testing. All families reported herein agreed to share clinical information and were enrolled under a research protocol approved by the Institutional Review Board of Baylor College of Medicine. Medical records were reviewed, and the referring providers were asked to complete a clinical questionnaire. All families who shared photographs signed consent for publication.

\section{Generation of patient fibroblast cell lines}

Individuals 13 and 14, who had a probable pathogenic variant affecting the start codon of NR2F1, had a skin biopsy performed to establish a fibroblast culture. Individual 13 consented to participate in a research protocol approved by the Institutional Review Board of Baylor College of Medicine. Individual 14 had a skin biopsy performed in 2004 for diagnostic purposes, and fibroblasts were frozen at that time. Upon receipt of signed consent from the individual's parents, fibroblasts were transferred to Dr Schaaf's laboratory for further testing.

\section{Tissue culture}

HEK293T cellswerepurchased from ATCC(Manassas, VA). Control human fibroblast cells and human lymphoblast cells were from the cell culture core at the Baylor College of Medicine Intellectual and Developmental Disabilities Research Center. HEK293T cells and human fibroblast cells were grown in Dulbecco's modified Eagle medium (Corning Cellgro, Manassas, VA). Human lymphoblast cells were grown in RPMI 1640 medium (Invitrogen, Waltham, MA). Both media were supplemented with $10 \%$ fetal bovine serum and $1 \%$ penicillin-streptomycin (Invitrogen, Waltham, MA). All cell cultures were maintained at $37^{\circ} \mathrm{C}$ in a humidified incubator supplemented with $5 \%$ carbon dioxide.

\section{Dual-luciferase reporter assay}

Three expression vectors were used in the assay. The pXP2-NGFI-A vector expresses firefly luciferase under control of the NR2F1-activated promoter, NGFI-A $(-168 /+33)$, kindly provided by Ming-Jer Tsai. ${ }^{10}$ The pRL-TK vector expresses Renilla luciferase as an internal control reporter and was purchased from Promega. Both the pXP2-NGFI-A and pRL-TK vectors were cotransfected with expression plasmid pcDNA5 of either wild-type or mutant mouse Nr2f1 into HEK293T cells. All point mutations were generated by a QuikChange sitedirected mutagenesis kit (Agilent, Santa Clara, CA). HEK293T cells were seeded in a 24 -well plate $24 \mathrm{~h}$ before transfection. Cells were cotransfected with $5 \mathrm{ng}$ of each expression vector (total of three) per well with Lipofectamine 2000 following the manufacturer's instructions. Cells were harvested $48 \mathrm{~h}$ after transfection, and the luciferase activity was measured following the manufacturer's instructions (Promega, Fitchburg, WI). The luciferase activity was quantified by normalizing firefly luciferase reads to Renilla luciferase reads. The luciferase activity of wild-type Nr2f1 and the empty vector was set as 100 and 0\%, respectively. Results are mean values \pm SEM from three independent experiments performed in triplicate.

\section{Western blot analysis}

Human patient or control fibroblast cell lines were harvested $\left(1.3 \times 10^{6}\right.$ cells $)$ and lysed with modified radioimmunoprecipitation assay buffer ( $25 \mathrm{mmol} / \mathrm{l}$ Tris- $\mathrm{HCl}$ (pH 7.8), $150 \mathrm{mmol} / \mathrm{l}$ $\mathrm{NaCl}, 1 \% \mathrm{NP}-40,1 \%$ sodium deoxycholate, $0.1 \%$ sodium dodecyl sulfate, and complete protease inhibitor cocktail (Roche, Indianapolis, IN)). Protein lysate was collected after boiling for $5 \mathrm{~min}$, followed by centrifugation at $13,000 \mathrm{rpm}$ for $10 \mathrm{~min}$. Proteins were quantified using a Pierce BCA Protein Assay Kit (Thermo Scientific, Waltham, MA). Protein (15 $\mu \mathrm{g})$ was resolved by electrophoresis on a NuPAGE $4-12 \%$ bis-Tris Gel (Life Technologies, Waltham, MA) and transferred to nitrocellulose membranes. Blots were blocked in $5 \%$ skim milk and incubated with anti-COUP-TFI antibody (1:1,000 dilution; catalog no. 6364S; Cell Signaling Technologies, Beverly, 
MA) or anti-GAPDH (1:20,000 dilution; catalog no. 2-RGM2; Advanced ImmnuoChemical, Long Beach, CA). Results are mean values \pm SEM from two independent control human fibroblast cell lines/two patient fibroblast cell lines (individuals 13 and 14) in one experiment.

\section{Quantitative real-time polymerase chain reaction}

Total RNA was extracted from either human lymphoblast or fibroblast cell lines with an miRNeasy Mini Kit (Qiagen, Valencia, CA) following the manufacturer's instructions. RNA was quantified using a NanoDrop 1000 spectrophotometer (Thermo Fisher, Waltham, MA). RNA was reverse-transcribed to complementary DNA using a reverse transcription kit (Qiagen, Valencia, CA). Complementary DNA samples then underwent quantitative real-time polymerase chain reaction on the CFX96 Touch Real-Time PCR Detection System (Bio-Rad Laboratories, Hercules, CA) with SYBR Green FastMix (Quanta Biosciences, Gaithersburg, MD). Relative quantities of NR2F1 messenger RNA were measured with the $\triangle \triangle \mathrm{CT}$ method and normalized against the housekeeping gene $G A P D H$. The quantitative real-time polymerase chain reaction primer sequences were as follows: human NR2F1 forward, 5'-ATCGTGCTGTTCACGTCAGAC-3'; humanNR2F1 reverse, 5'-TGGCTCCTCACGTACTCCTC-3'; human GAPDH forward, 5'-CGACCACTTTGTCAAGCTCA-3'; human GAPDH reverse, 5'-TTACTCCTTGGAGGCCATGT-3'. Results are mean values \pm SEM from two independent control human lymphoblast cell lines/two independent control human fibroblast cell lines/two patient fibroblast cells lines (individuals 13 and 14) in one experiment.

\section{RESULTS}

We identified 20 individuals with novel pathogenic NR2F1 variants (seven missense, five translation initiation variants, two frameshifting insertion/deletions (indel), one nonframeshifting indel, and five whole-gene deletions).

\section{Enrichment of pathogenic NR2F1 missense variants in the DNA-binding domain and the ligand-binding domain} Six missense variants (individual 1, p.Cys128Arg; individual 2, p.Arg135Ser; individual 3, p.Cys138Tyr; individual 4, p.Arg142Leu; individual 5, p.Cys146Arg; individual 7, p.Gly368Asp) were predicted to be pathogenic by two widely used variant annotation tools, Mutation Taster and PolyPhen-2 (see Supplementary Table S1 online). To study the functional effects of these mutations in NR2F1, we performed a dual-luciferase reporter assay. In this assay, firefly luciferase was under control of an NR2F1-activated promoter, NGFI-A $(-168 /+33),{ }^{10}$ and HEK293T cells were cotransfected with this plasmid with either wild-type or mutant Nr2f1 constructs. The second luciferase, Renilla, served as an internal control for normalization. As shown in Figure $\mathbf{1 b}$, all six missense variants significantly impaired the ability of NR2F1 to fully activate the NGFI-A $(-168 /+33)$ reporter. Five of them reside in the DBD and one is located in the ligand-binding domain (Figure 1a) of NR2F1. One additional missense variant was identified (individual 6,

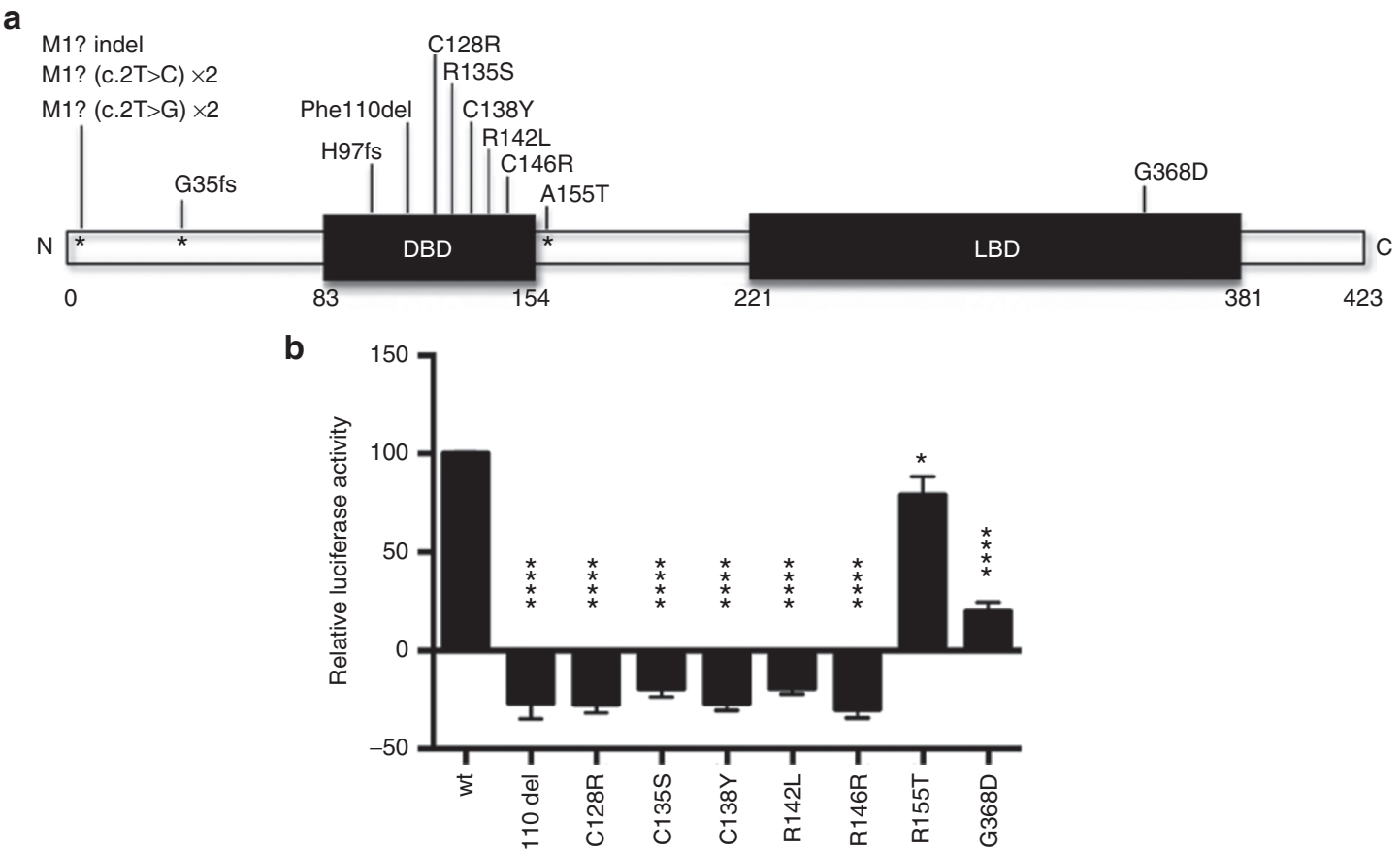

Figure 1 Pathogenic NR2F1 variants decrease transcriptional activity. (a) Distribution of NR2F1 point mutations in patients with Bosch-Boonstra-Schaaf optic atrophy syndrome. Most of the mutations (except p.Ala155Thr) fall into the DNA-binding domain or ligand-binding domain. The number of individuals who share the same translation initiation variant is indicated. (b) In vitro dual-luciferase reporter assay for NR2F1 point mutations. HEK293T cells cotransfected with dual-luciferase vectors (pXP2-NGFI-A and pRL-TK vectors) and either wild-type or mutant Nr2f1 expression vectors were analyzed. The luciferase activity of wild-type $\mathrm{Nr} 2 \mathrm{f1}$ and the empty vector are set as 100 and $0 \%$, respectively. Data are means $\pm \operatorname{SEM}(n=3)$. ${ }^{\star} P<0.05, * \star \star \star P<0.0001$. 
p.Ala155Thr), which is located outside of, but adjacent to, the DBD. This variant was predicted to be pathogenic by Mutation Taster but not PolyPhen-2. The luciferase assay showed that this mutation significantly decreased reporter activity, but only by $30 \%$, which is less dramatic than the decrease in activity resulting from missense substitutions in the DBD itself. Finally, we confirmed that one nonframeshifting indel within the DBD (individual 8, p.Phe110del) also reduced reporter activity. Collectively, the enrichment of pathogenic mutations in the DBD and ligand-binding domain indicates the importance of these highly conserved functional domains in NR2F1.

\section{Decreased NR2F1 protein expression in translation initiation mutations}

Coding nonsynonymous variants of the translation initiation codon of $N R 2 F 1$ have not been reported to date. In this study, we identified five individuals with variants of the translation initiation codon. Two unrelated individuals (individuals 11 and 12) shared the same mutation (c.2T>G, p.M1?). Two additional and unrelated individuals (individuals 13 and 14) shared another variant, c.2T $>$ C, p.M1?. One individual (individual 15) carried a nonframeshifting indel affecting the start codon (c.2_4delTGGinsGGA, p.M1?). Given that the third codon of NR2F1 is "ATG," which could potentially serve as an alternative start codon when the original start codon is mutated, it was difficult to predict whether coding nonsynonymous changes of M1 would affect translation and thus the abundance of the NR2F1 protein. To test this, we first compared NR2F1 expression levels in human lymphoblast and skin fibroblast cell lines, two easily accessible peripheral cell types of living individuals. We showed that human fibroblasts have much higher NR2F1 expression than lymphoblasts (see Supplementary Figure S1 online). We were able to generate fibroblast cell lines using cells from individuals 13 and 14 . The abundance of NR2F1 protein is decreased by approximately $60 \%$ in each of these individuals when compared with control cell lines (Figure 2a). To determine whether decreased NR2F1 protein levels in patient fibroblasts may also result from altered transcription, we assessed NR2F1 messenger RNA levels and compared those with levels in unaffected controls. We found that NR2F1 messenger RNA is approximately $45 \%$ decreased in both patients' fibroblast cell lines (Figure $2 \mathbf{b}$ ), suggesting that the reduction of NR2F1 protein due to genetic variation in the translation initiation codon may be the result of alterations at both the translational and transcriptional levels.

\section{Clinical phenotypes of NR2F1 haploinsufficiency}

Optic atrophy, developmental delay, and intellectual disability are the most well-established clinical features of individuals with BBSOAS. ${ }^{1}$ In this study, we report 20 new individuals with heterozygous $N R 2 F 1$ variants, including seven missense changes, five translation initiation variants, two frameshifting indels, one nonframeshifting indel, and five whole-gene deletions (Table 1). The size of the NR2F1 deletions in this study ranges from 0.2 to $5 \mathrm{Mb}$, with $0.2 \mathrm{Mb}$ being the smallest a
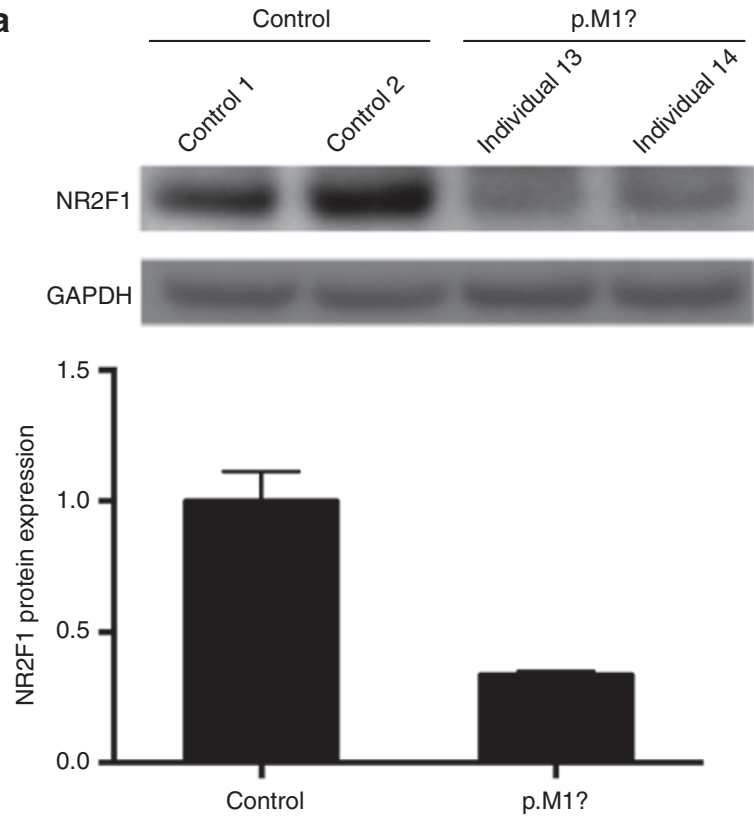

b

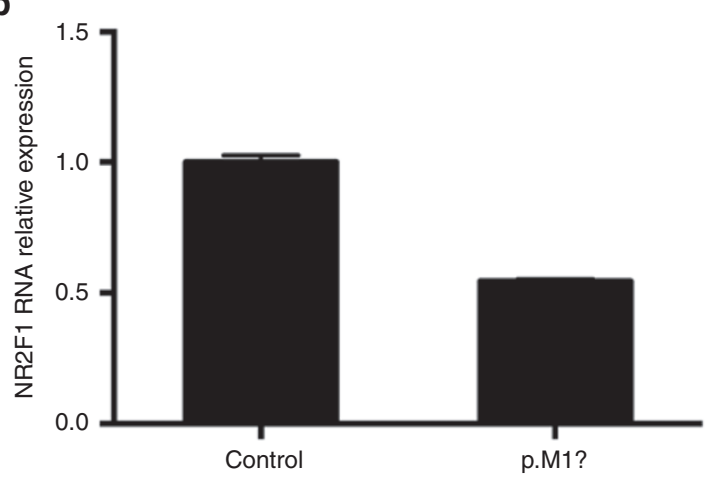

Figure 2 The translation initiation mutation decreases NR2F1 protein and messenger RNA (mRNA) level. (a) Protein quantification of NR2F1 from human fibroblast cell lines of control individuals or carriers of the translation initiation variant (from individuals 13 and 14). Data are normalized to GAPDH protein levels. Data are mean $\pm \operatorname{SEM}(n=2)$. (b) NR2F1 mRNA quantification by quantitative reverse transcriptase-polymerase chain reaction from human fibroblast cell lines of control individuals or carriers of the translation initiation variant (from individuals 13 and 14). Data are normalized to GAPDH mRNA levels. Data are mean $\pm \operatorname{SEM}(n=2)$.

deletion reported to date (Figure 3). Two individuals with a $0.9-\mathrm{Mb}$ deletion (individuals 17 and 18) are father and son, thereby representing the first familial case of a pathogenic NR2F1 variant. In this study 95\% (19/20) of the individuals manifest developmental delay/intellectual disability, and the majority (17/20) have substantial visual impairment, among them 15 with confirmed optic atrophy (Table 1). Hypotonia is present in $75 \%(15 / 20)$, and $60 \%(12 / 20)$ have a history of oromotor dysfunction, including problems with sucking, chewing, swallowing, and feeding. Brain magnetic resonance imaging had been performed on 15 of the 20 individuals and indicated thinning of the corpus callosum in 53\% (8/15). Interestingly, NR2F1 haploinsufficiency also seemed to alter the behaviors of the affected individuals. Repetitive behaviors were reported in $40 \%(8 / 20)$, and $35 \%(7 / 20)$ had received a 


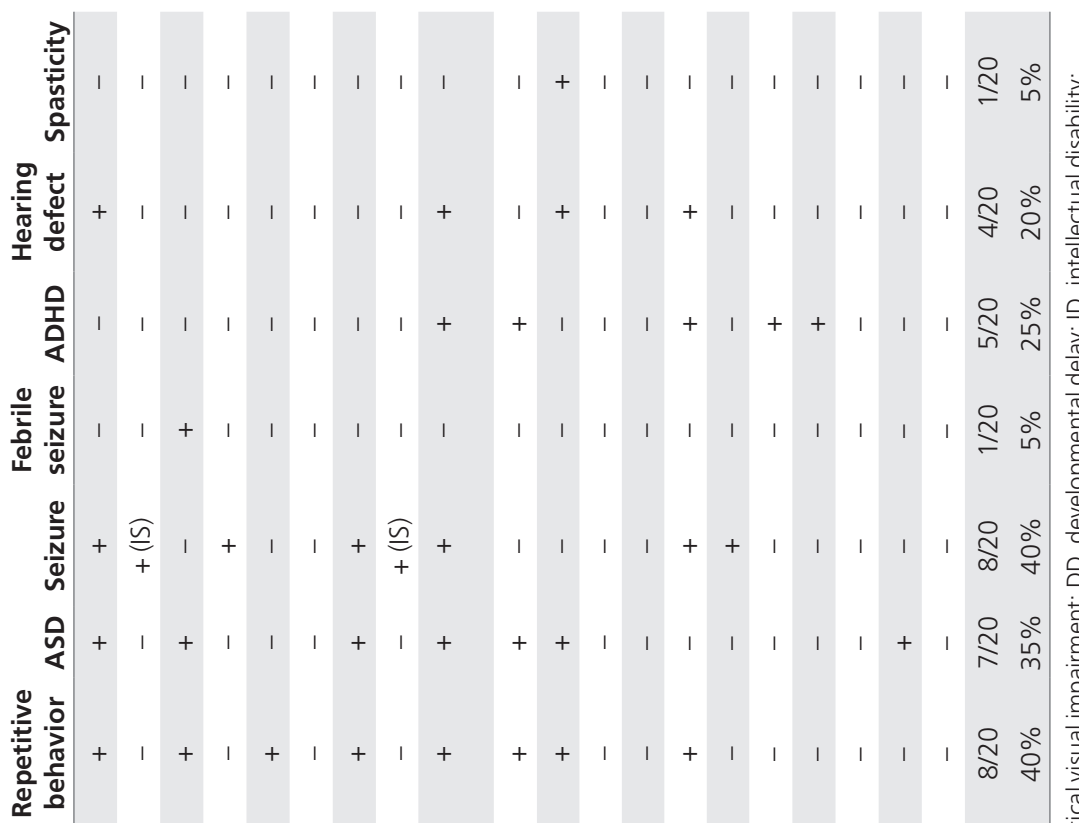

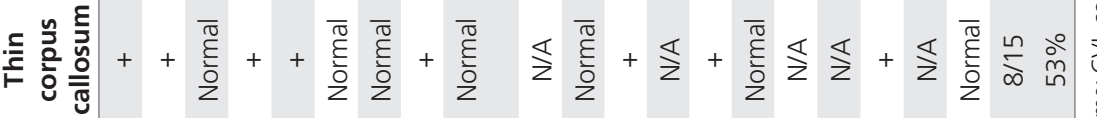

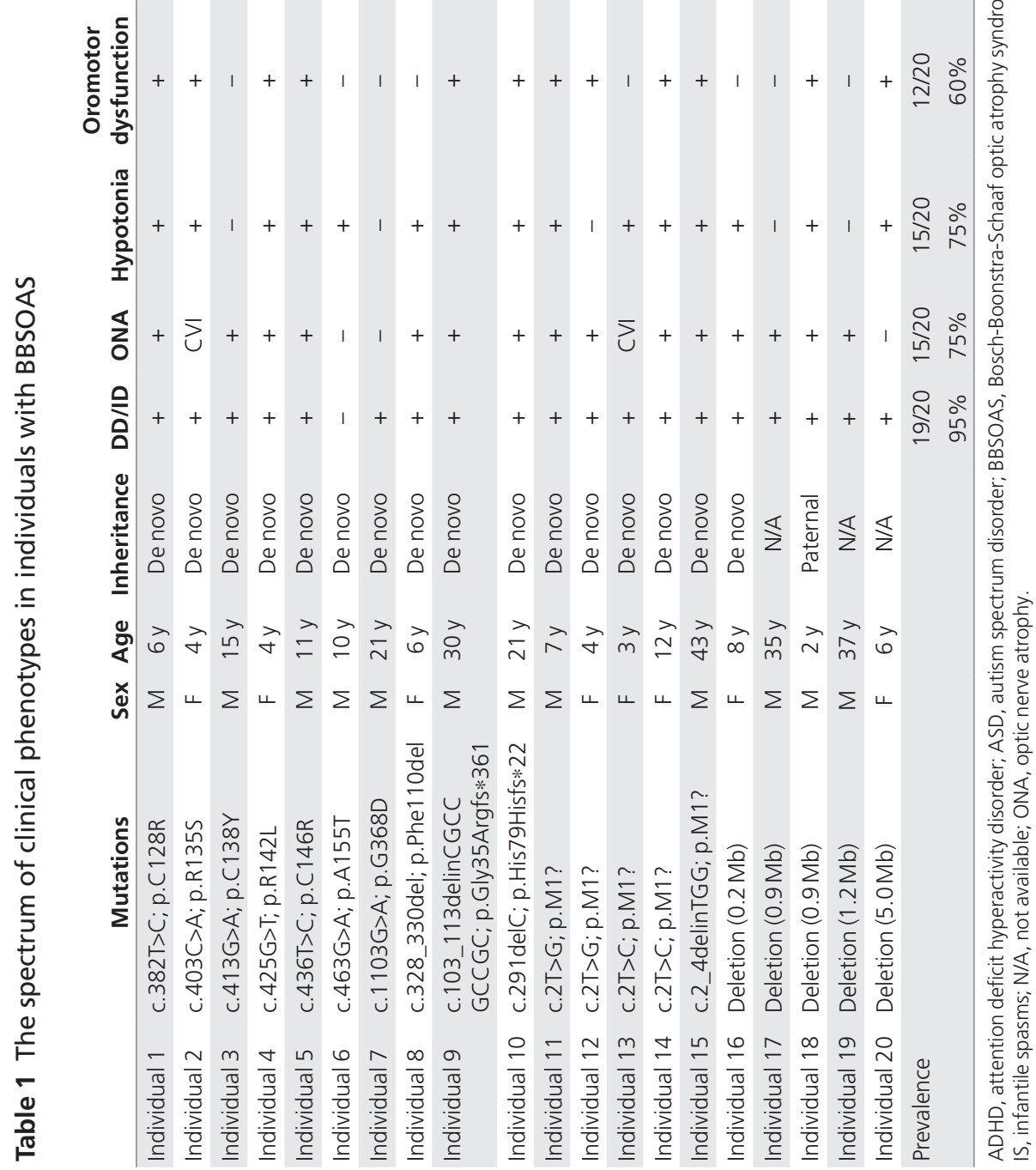



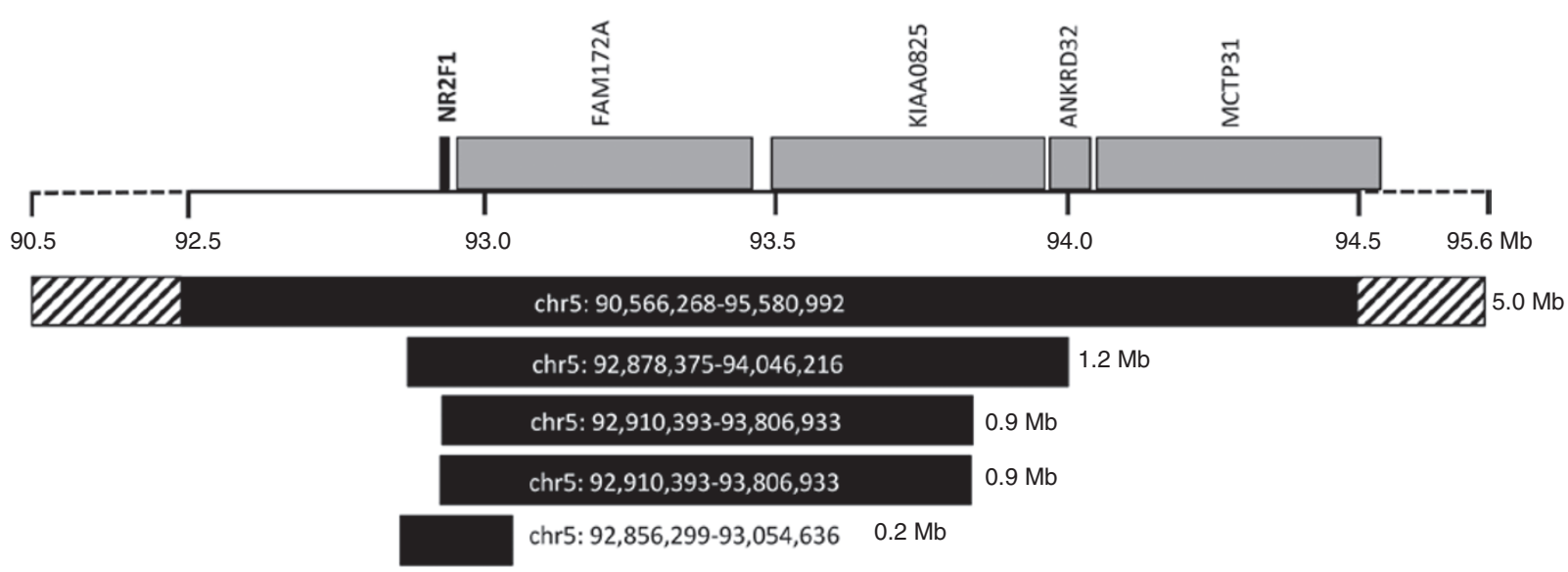

Figure 3 Overview of the microdeletions encompassing the NR2F1 gene from patients with Bosch-Boonstra-Schaaf optic atrophy syndrome. The sizes of the microdeletions range from 0.2 to $5.0 \mathrm{Mb}$. Individuals 17 and 18 are a father and son who share the same microdeletion containing NR2F1.
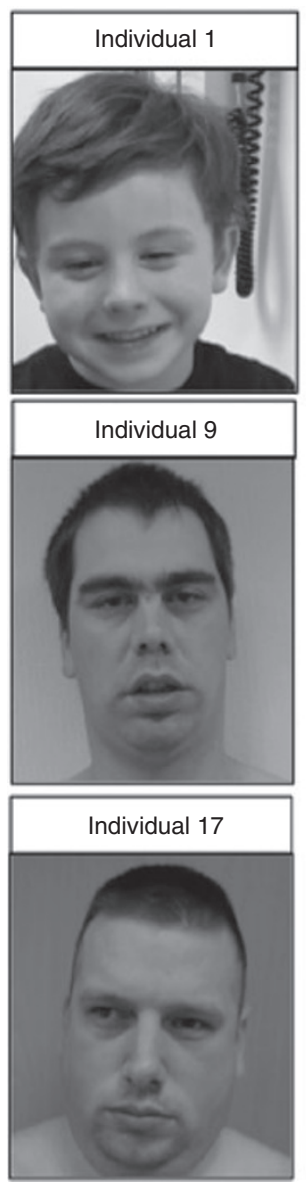

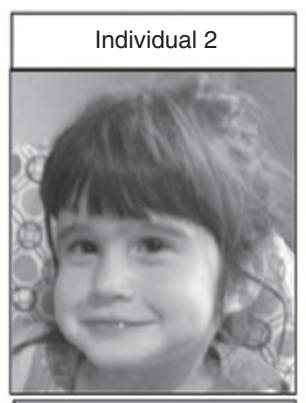

Individual 10
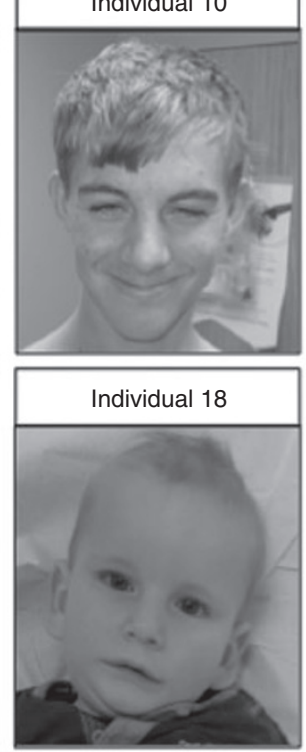
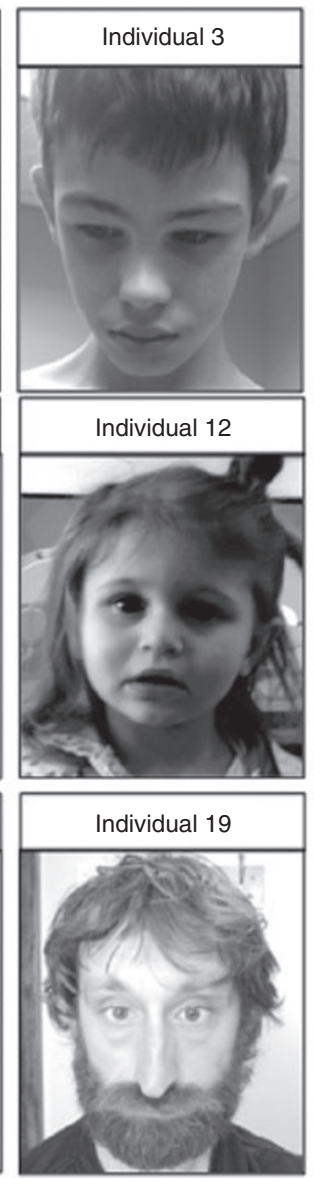
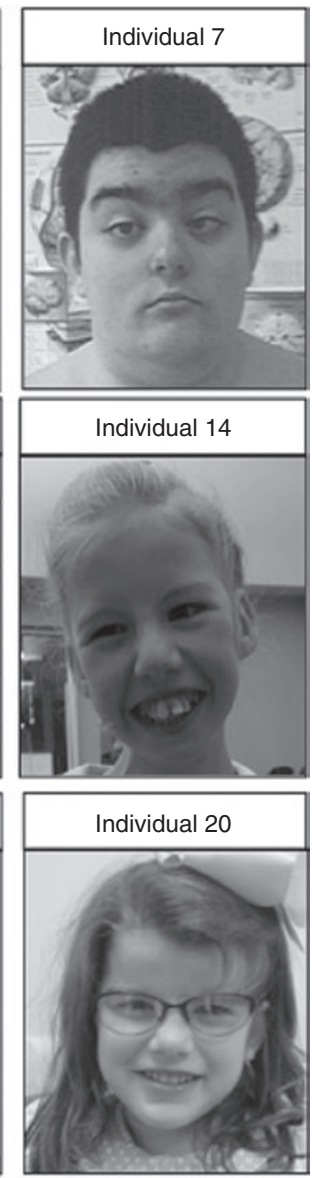

Figure 4 Facial appearance of patients with Bosch-Boonstra-Schaaf optic atrophy syndrome. While some individuals manifest mild dysmorphic facial features, they do not appear to be consistent between individuals.

diagnosis of autism spectrum disorder. A history of seizures was present in $40 \%$ (8/20); two of these eight individuals had a history of infantile spasms. In addition, one individual had had a single febrile seizure. Other clinical features were present at lower prevalences, including 25\% (5/20) with attention deficit hyperactivity disorder, $20 \%(4 / 20)$ with hearing impairment, and 5\% (1/20) with spasticity. While some individuals manifest mild dysmorphic facial features, they do not seem to be consistent between individuals (Figure 4). In summary, we showed that NR2F1 haploinsufficiency leads not only to cognitive deficits and visual impairment but also to a much broader range of phenotypic features, including 
low muscle tone, aberrant behavior, and structural changes of brain morphology.

\section{DISCUSSION}

The large number of individuals with NR2F1 haploinsufficiency reported herein allows us to better characterize the phenotypic spectrum of BBSOAS. One of the emerging phenotypes associated with heterozygous pathogenic NR2F1 variants is epilepsy. In fact, $N R 2 F 1$ has recently been reported in the context of infantile spasms. ${ }^{15-17}$ In our study, individuals 2 and 8 were diagnosed with infantile spasms, another six individuals were reported as having other forms of epilepsy (generalized tonic-clonic, atonic, and focal seizures; see Supplementary Information online), and individual 3 had experienced a single febrile seizure. Hearing impairment had previously been reported in one individual with an NR2F1 deletion, ${ }^{12}$ but not in other haploinsufficient individuals. ${ }^{1}$ Here we report that 4 of 20 individuals have hearing loss, indicating reduced penetrance $(20 \%)$ for this phenotype. Based on these observations, we suggest that BBSOAS can manifest a wide array of clinical features, but developmental delay/intellectual disability and vision impairment caused by optic atrophy remain the most prevalent ones.

We established a luciferase reporter assay, which provides a valuable functional tool to evaluate the effects of NR2F1 variants on transcriptional activity. ${ }^{1}$ In this study we found that all missense variants within the DBD of NR2F1 (p.Cys128Arg, p.Arg135Ser, p.Cys138Tyr, p.Arg142Leu, and p.Cys146Arg) completely abolish transcriptional activity. Only two of the six missense variants identified here do not completely abolish transcriptional activity in the luciferase assay: p.Ala155Thr (individual 6), which is located immediately adjacent to the DBD, and p.Gly368Asp (individual 7), in the ligand-binding domain of NR2F1. Interestingly, the clinical phenotypes of these individuals are relatively mild when compared with those of others with BBSOAS. They both have only mild visual impairment, and no optic atrophy is present. In addition, individual 6, who has the functionally mildest variant, met his developmental milestones on time and currently, at the age of 10 years, attends regular education classes. However, he does have a history of speech problems and dysarthria. Although we currently do not have a large enough number of individuals with hypomorphic NR2F1 variants, this study provides a first hint that genotype-phenotype correlations may exist in the context of BBSOAS. We hope that the identification of additional individuals will establish more robust genotype-phenotype correlations in the future.

We identified five individuals with variants of the translation initiation codon. Based on fibroblast cell lines from two of the five individuals, we showed that these lead to NR2F1 protein reduction, which seems to be the result of alterations at both translational and transcriptional levels. Although the effects of alternate start codons on gene expression are poorly understood, one previous study showed that alternate start codon usage decreases the efficiency of the gene expression at the transcriptional level. ${ }^{18}$ The detailed mechanisms of how translation initiation codon variants affect NR2F1 gene expression definitely require further investigation.

To our surprise, the five missense variants in the DBD (p.Cys128Arg, p.Arg135Ser, p.Cys138Tyr, p.Arg142Leu, and p.Cys146Arg) led to decreased reporter activities relative to the empty vector negative control (see negative value of luciferase activity in Figure 1b). This raises the intriguing question of whether a dominant negative effect may play a role in BBSOAS, given that NR2F1 binds to DNA in the form of dimers. ${ }^{19}$ In other words, individuals with heterozygous whole-gene deletions might generally have milder phenotypes compared with those who carry a heterozygous deleterious missense mutation in NR2F1. Indeed, the five individuals with NR2F1 deletion (individuals 16-20) in this study do have a lower prevalence of the majority of clinical phenotypes when compared with those who carry NR2F1 missense variants that completely abolish transcriptional activity (individuals $1-5$ ). These clinical features include hypotonia (60 vs. $80 \%$ ), oromotor dysfunction (40 vs. $80 \%$ ), thin corpus callosum (50 vs. $80 \%$ ), repetitive behaviors (0 vs. $60 \%$ ), autism spectrum disorder ( 20 vs. $40 \%$ ), seizures ( 0 vs. $60 \%$ ), and hearing defect ( 0 vs. $20 \%$ ). The absence of optic atropy in individual 20, who is one of the only three individuals without significant visual impairment in this study, may be another example of a milder phenotype with a whole-gene deletion.

The physiological functions of $N r 2 f 1$ have been studied extensively in mice. While most, if not all, studies have focused on homozygous knockout animals, and all reported humans diagnosed with BBSOAS carry heterozygous pathogenic NR2F1 variants, the murine studies may inform us, at least partially, about some pathomechanisms underlying specific clinical phenotypes. Constitutive knockout of $\mathrm{Nr} 2 \mathrm{f1}$ is perinatally lethal in mice. Most homozygous knockout mice die within the first 2 days of life as a result of starvation and dehydration. ${ }^{9}$ Defects in the formation of the glossopharyngeal nerve in these $\mathrm{Nr}_{2} \mathrm{f1}^{-{ }^{-/}}$ pups have been proposed to account for that outcome, and this observation may relate to the oromotor dysfunction observed in heterozygous human individuals. Abnormal development of the corpus callosum, the hippocampal commissure, and thalamocortical projections is seen in embryonic Nr2f1 ${ }^{-1-}$ brains. ${ }^{8,20}$ The presence of dysplasia or hypoplasia of the corpus callosum in $53 \%$ of individuals who had clinical magnetic resonance imaging suggests that abnormalities of neuronal guidance and establishment of fiber tracts in the central nervous system may also occur in patients with heterozygous BBSOAS. Several other clinical features appreciated in the human patients-for example, intellectual disability, autism spectrum disorder, and seizures-will be challenging to study in a mouse model, given the perinatal lethality of the homozygous knockout. Whether the $\mathrm{Nr}_{2} \mathrm{fl}^{-/+}$mouse is an adequate model to recapitulate human phenotypes has yet to be shown.

In summary, we expand the clinical phenotypic spectrum associated with BBSOAS, and we show how functional testing can be useful to inform us about the pathogenic significance of novel variants in the NR2F1 gene. 
As the number of individuals identified with BBSOAS continues to grow, families are beginning to connect via social media (https://www.facebook.com/pages/NR2F1-Collabora tive/434582583373135). To gain further insight into the phenotypic spectrum of BBSOAS, a Web-based database has been developed so that the phenotypes of individuals with NR2F1 aberrations can be updated (http://www.nr2flgene.com).

\section{SUPPLEMENTARY DATA}

Supplementary information is linked to the online version of the paper at http://www.nature.com/gim

\section{ACKNOWLEDGMENTS}

C.P.S. is generously supported by the Joan and Stanford Alexander family. C.P.S. has received a Clinical Scientist Development Award from the Doris Duke Charitable Foundation. R.A.L. is a senior scientific investigator for Research to Prevent Blindness, whose unrestricted funds to his department support part of these studies. This research is supported by the Intellectual and Developmental Disabilities Research Center (1U54 HD083092), Stichting ODAS (to F.N.B. and F.P.M.C.), Vereniging Bartiméus-Sonneheerdt (5781251 to F.N.B. and F.P.M.C.), Oogfonds (to F.P.M.C., F.N.B., and B.B.A.deV.), and LSBS (to F.P.M.C., F.N.B., and B.B.A.deV.). The authors are grateful to the individuals and their families for their support and for participating in our research study. They thank Ming-Jer Tsai and Mafei Xu for providing technical assistance and Huda Y. Zoghbi for valuable input, guidance, and discussion.

\section{DISCLOSURE}

W.K.C. is a consultant for BioReference Laboratories. The other authors declare no conflict of interest.

\section{REFERENCES}

1. Bosch DG, Boonstra FN, Gonzaga-Jauregui C, et al.; Baylor-Hopkins Center for Mendelian Genomics. NR2F1 mutations cause optic atrophy with intellectual disability. Am J Hum Genet 2014;94:303-309.

2. Sagami I, Tsai SY, Wang H, Tsai MJ, O'Malley BW. Identification of two factors required for transcription of the ovalbumin gene. Mol Cell Biol 1986;6: 4259-4267.

3. Lin FJ, Qin J, Tang K, Tsai SY, Tsai MJ. Coup d'Etat: an orphan takes control. Endocr Rev 2011;32:404-421.
4. Faedo A, Tomassy GS, Ruan Y, et al. COUP-TFI coordinates cortical patterning, neurogenesis, and laminar fate and modulates MAPK/ERK, AKT, and betacatenin signaling. Cereb Cortex 2008;18:2117-2131.

5. Armentano M, Chou SJ, Tomassy GS, Leingärtner A, O'Leary DD, Studer M. COUP-TFI regulates the balance of cortical patterning between frontal/motor and sensory areas. Nat Neurosci 2007;10:1277-1286.

6. Zhou C, Tsai SY, Tsai MJ. COUP-TFI: an intrinsic factor for early regionalization of the neocortex. Genes Dev 2001;15:2054-2059.

7. Alfano C, Magrinelli E, Harb K, Hevner RF, Studer M. Postmitotic control of sensory area specification during neocortical development. Nat Commun 2014;5:5632.

8. Zhou C, Qiu Y, Pereira FA, Crair MC, Tsai SY, Tsai MJ. The nuclear orphan receptor COUP-TFI is required for differentiation of subplate neurons and guidance of thalamocortical axons. Neuron 1999;24:847-859.

9. Qiu Y, Pereira FA, DeMayo FJ, Lydon JP, Tsai SY, Tsai MJ. Null mutation of mCOUP-TFI results in defects in morphogenesis of the glossopharyngeal ganglion, axonal projection, and arborization. Genes Dev 1997;11:1925-1937.

10. Tang K, Xie X, Park JI, Jamrich M, Tsai S, Tsai MJ. COUP-TFs regulate eye development by controlling factors essential for optic vesicle morphogenesis. Development 2010;137:725-734.

11. Satoh S, Tang K, lida A, et al. The spatial patterning of mouse cone opsin expression is regulated by bone morphogenetic protein signaling through downstream effector COUP-TF nuclear receptors. J Neurosci 2009;29: 12401-12411.

12. Brown KK, Alkuraya FS, Matos M, Robertson RL, Kimonis VE, Morton CC. NR2F1 deletion in a patient with a de novo paracentric inversion, inv(5) (q15q33.2), and syndromic deafness. Am J Med Genet A 2009;149A: 931-938.

13. Al-Kateb H, Shimony JS, Vineyard M, Manwaring L, Kulkarni S, Shinawi M. NR2F1 haploinsufficiency is associated with optic atrophy, dysmorphism and global developmental delay. Am J Med Genet A 2013;161A:377-381.

14. Bosch DG, Boonstra FN, de Leeuw N, et al. Novel genetic causes for cerebral visual impairment. Eur J Hum Genet; e-pub ahead of print 9 September 2015.

15. Michaud JL, Lachance M, Hamdan FF, et al. The genetic landscape of infantile spasms. Hum Mol Genet 2014;23:4846-4858.

16. Hino-Fukuyo N, Kikuchi A, Arai-Ichinoi N, et al. Genomic analysis identifies candidate pathogenic variants in 9 of 18 patients with unexplained West syndrome. Hum Genet 2015;134:649-658.

17. Dimassi $S$, Labalme $A$, Ville $D$, et al. Whole-exome sequencing improves the diagnosis yield in sporadic infantile spasm syndrome. Clin Genet 2016;89:198204.

18. Panicker IS, Browning GF, Markham PF. The effect of an alternate start codon on heterologous expression of a phoA fusion protein in Mycoplasma gallisepticum. PLoS One 2015;10:e0127911.

19. Cooney AJ, Tsai SY, O'Malley BW, Tsai MJ. Chicken ovalbumin upstream promoter transcription factor (COUP-TF) dimers bind to different GGTCA response elements, allowing COUP-TF to repress hormonal induction of the vitamin D3, thyroid hormone, and retinoic acid receptors. Mol Cell Biol 1992;12:4153-4163.

20. Armentano M, Filosa A, Andolfi G, Studer M. COUP-TFI is required for the formation of commissural projections in the forebrain by regulating axonal growth. Development 2006;133:4151-4162. 